\title{
Bi-Polar RF, Still the Gold Standard for Non-Invasive Fat Volume Reduction
}

\author{
James K. M. Chan1, Inna Belenky², Monica Elman ${ }^{3 *}$ \\ ${ }^{1}$ DR JAMES CLINIC, Hong Kong, China \\ ${ }^{2}$ Viora Inc., Jersey City, NJ, USA \\ ${ }^{3}$ Elman Medical Services Ltd., Tel Aviv, Israel \\ Email: *monica@elman.co.il
}

Received 2 September 2015; accepted 20 November 2015; published 23 November 2015

Copyright (C) 2015 by authors and Scientific Research Publishing Inc.

This work is licensed under the Creative Commons Attribution International License (CC BY). http://creativecommons.org/licenses/by/4.0/

(c) ()

\section{Abstract}

Background and Objective: Non-invasive body contouring treatments is one of the fastest growing markets in the aesthetic field. The main purpose of radiofrequency (RF)-based devices for body contouring is to produce thermal stimulus which leads to skin tightening effect by dermal collagen production and decreases the volume of adipose tissue by a reduction of the adipocyte's cytosolic. The cutaneous application of RF devices in Asian patients differs in several respects from their use in Caucasians; therefore the objective of this study was to evaluate the safety and efficacy of a novel RF device used for focal fat reduction. Materials and Methods: Twenty-two Asian patients aged 24 - 51, Fitzpatrick skin types III-V, were enrolled to the study and received 6 sessions of consecutive abdomen contouring treatment with bi-polar RF device combined with a mechanical pressure. Statistical linear correlation tests and descriptive analysis were performed on the cohort. Results: All twenty-two patients treated for six treatments showed some degree of circumferential reduction with a stable weight. No adverse events were recorded during the whole period of trial. During the 3-month follow up visit, no deterioration of the body condition was detected. No statistical significant relation was found between circumference change and weight, age, height or BMI. Conclusions: The majority of the patients exhibited a substantial circumferential reduction, not at the expense of weight loss. The bi-polar RF device was found to be safe for Asian skin.

\section{Keywords}

Adipose Tissue, Asian Patients, Radiofrequency, Vacuum

\section{Introduction}

Non-invasive body contouring treatments is one of the fastest growing markets in the aesthetic field [1]. These "Corresponding author.

How to cite this paper: Chan, J.K.M., Belenky, I. and Elman, M. (2015) Bi-Polar RF, Still the Gold Standard for Non-Invasive Fat Volume Reduction. Journal of Cosmetics, Dermatological Sciences and Applications, 5, 247-253. 
treatments have grew from mechanical rollers and suction technologies, to thermal based technologies including radio-frequency (RF) energy, infrared light, high intensity focused ultrasound energy (HIFU), and for nonthermal technologies such as cryolipolysis, pulsed focused ultrasound energy, low-level laser therapy (LLLT), etc. [2]-[10]. In some devices the thermal heat is combined with mechanical pressure technology [2]-[6] [11]. The mechanical pressure stimulates the lymphatic flux which reduces edema and toxic environment.

Main purposes of RF-based devices are skin tightening and decrease the volume of adipose tissue. Skin is tightened by immediate collagen contraction, subsequent collagen production and remodeling. Reduction of the adipocyte's cytosolic volume by normal lipolysis without direct cellular destruction is also obtained due to heat generation in the tissue. The lipolysis process is a normal metabolism of the adipose tissue through stimulation of epinephrine mediated membrane bound lipase enzyme. During the lipolysis process the triglycerides (the main cytosolic volume) are converted (hydrolyzed) to glycerol and free fatty acids. These free fatty acids and glycerol egress out of the cell and are mobilized and metabolized according to body's needs [2].

The cutaneous application of RF devices in Asian patients differs in several respects from their use in Caucasians [12]. But, since RF energy is color-blind technology, the response of the Asian population to the body contouring treatments is assumed to be similar to the Caucasian Population. The objective of this study was to evaluate the safety and efficacy of a novel RF-based device used for focal fat reduction.

\section{Materials and Methods}

\subsection{Patient's Specifications}

Twenty-two Asian patients from Hong Kong (16 females and 6 males) aged 24 - 51 (average 39.59 years, SD 7.14 years), Fitzpatrick skin types III-V (according to 6 types Fitzpatrick phototyping scale), were enrolled to the study after informed consent was obtained. The patients selected according to the following inclusion criteria: 1) no contraindication for the RF-based treatment; 2) age below 18 years old; 3) BMI 18.5 - 39.9; 4) realistic treatment expectations.

The height range of patients deployed between $152-180 \mathrm{~cm}$ (average $166.27 \mathrm{~cm}$, SD $7.92 \mathrm{~cm}$ ) with initial body weight of 49 - $114.5 \mathrm{Kg}$ (average $63.36 \mathrm{Kg}$, SD $14.63 \mathrm{Kg}$ ) and calculated BMI (Body Mass Index) of 18 36 (average 22.78, SD 4.06). Seventeen patients were in range of "normal" (healthy weight), one patient was in the "underweight" category, with BMI = 18.0 (patient No. 21), three patients were in "overweight" category (patient No. 1, 17 and 19) and one patient was in "obese class I" (moderately obese) category (patient No. 18) (Table 1).

All patients, except patients No. 18 and 21, had maintained a stable weight (weight fractionations were limited to $\pm 2 \mathrm{Kg}$ ) during the whole period of the trial (Table 1). Patients presented similar focal fat accumulation on the abdomen area. No patient had undergone any previous treatments and/or medications for fat volume reduction.

\subsection{Treatment Device and Treatment Regime}

\subsubsection{RF-Based Device}

All twenty two patients were treated with bi-polar RF device (Viora Inc., Jersey City, NJ, USA) combined with a mechanical pressure. The system integrates CORETM technology, which allows independent heating depth control with three distinguished frequencies of RF energy: $0.8,1.7$ and $2.45 \mathrm{MHz}$, and an additional channel which combines all three frequencies in a single pulse [11].

\subsubsection{Treatment Applicator}

All patients were treated with B-Contour applicator which designed for treatment of large skin areas. This applicator emits vacuum-assisted pulsed RF power up to $50 \mathrm{~W}$ with a treatment spot size of $35 \times 30 \mathrm{~mm}$ [11].

\subsubsection{Treatment Area and Regime}

The entire study period was hold during 6 months, including the 3 months follow-up visits. All patients kept on their normal diet and level of exercise during treatment period. The patients received 6 consecutive sessions on the abdomen area with one week interval. The treatment procedure was based on "Body Contouring Protocol" designed in three phases: I) Pre-heating phase, II) adipose tissue heating phase and III) fibroblast stimulation 
Table 1. Patient characteristics.

\begin{tabular}{|c|c|c|c|c|c|}
\hline Patient No. & Age & Sex $^{*}$ & BMI & Weight Fluctuations (Kg) & Fitzpatrick Skin Type \\
\hline 1 & 38 & $\mathrm{~F}$ & 29.7 & 0.0 & IV \\
\hline 2 & 50 & M & 24.6 & 0.1 & $\mathrm{~V}$ \\
\hline 3 & 38 & $\mathrm{~F}$ & 24.8 & 0.0 & IV \\
\hline 4 & 51 & $\mathrm{~F}$ & 22.7 & 0.0 & $\mathrm{~V}$ \\
\hline 5 & 43 & $\mathrm{~F}$ & 20.1 & -2.0 & IV \\
\hline 6 & 38 & $\mathrm{~F}$ & 21.0 & -1.7 & III \\
\hline 7 & 29 & $\mathrm{~F}$ & 20.3 & -0.8 & III \\
\hline 8 & 38 & $\mathrm{~F}$ & 23.4 & 1.9 & III \\
\hline 9 & 46 & $\mathrm{~F}$ & 21.2 & 0.5 & IV \\
\hline 10 & 38 & M & 22.5 & -0.2 & $\mathrm{~V}$ \\
\hline 11 & 24 & F & 20.0 & -0.8 & III \\
\hline 12 & 46 & M & 21.9 & 0.3 & IV \\
\hline 13 & 31 & $\mathrm{~F}$ & 23.3 & -0.2 & III \\
\hline 14 & 27 & F & 21.4 & 0.7 & III \\
\hline 15 & 42 & F & 18.8 & 0.2 & III \\
\hline 16 & 43 & $\mathrm{~F}$ & 19.4 & 0.0 & IV \\
\hline 17 & 43 & $\mathrm{M}$ & 26.2 & 0.6 & III \\
\hline 18 & 46 & M & 36.1 & -11.8 & III \\
\hline 19 & 41 & M & 25.2 & 0.0 & IV \\
\hline 20 & 38 & $\mathrm{~F}$ & 19.2 & 0.6 & III \\
\hline 21 & 35 & F & 18.0 & 4.6 & III \\
\hline 22 & 46 & $\mathrm{~F}$ & 21.6 & -0.6 & III \\
\hline
\end{tabular}

F: Female. M: Male.

phase (skin tightening finishing) [11]. The skin temperature was continuously monitored at $39^{\circ} \mathrm{C}-42^{\circ} \mathrm{C}$, using IR (infrared) thermometer (Fluke 561, Everett, WA USA).

Each treatment session lasted about $20 \pm 2$ minutes in accordance with protocol guidelines.

\subsection{Clinical Measurements and Statistical Analysis}

\subsubsection{Clinical Measurements}

During the enrollment visit, patient's parameters including gender, age, height, weight, BMI and circumferential measurements were recorded before each treatment and in 3-month follow-up visit.

The weight fluctuations (in $\mathrm{Kg}$ ) were calculated as following: weight $(\mathrm{Kg})$ measured one week after the last treatment session minus weight $(\mathrm{Kg})$ measured in the baseline meeting. The BMI was calculated according to standard guidelines.

The circumferential measurements of the neck, arms, chest, upper abdomen, umbilicus, lower abdomen, hips, thighs and knees, were made using measuring tape, on constant points on each body part. The upper abdomen was determined as a part above the umbilicus and the lower abdomen, as part below. The "Total" circumferential measurement was calculated as a sum of all body parts circumferential measurements. The "Upper body" circumferential measurement was calculated as a sum of neck, arms, chest and upper abdomen circumferential measurements. The "Lower body" circumferential measurement was calculated as a sum of lower abdomen, hips, thighs and knees circumferential measurements. 
The circumferential change (in $\mathrm{cm}$ ) was calculated as following: circumference $(\mathrm{cm})$ recorded one week after the last session minus circumference $(\mathrm{cm})$ recorded in the baseline meeting.

Photography was taken in the baseline, one week after the $6^{\text {th }}$ session and one week after the last treatment session if a patient had more than 6 treatment sessions.

\subsubsection{Statistical Analysis}

Twenty patients were enrolled to the statistical analysis. Patients No. 18 and 21 were excluded due to unstable weight during the trial. These patients were also excluded when we enrolled only patients that are in range of average value of the initial patient's weight \pm 2 SD.

All statistical tests were performed using the software package for statistical science (SPSS for Windows, Version 14, SPSS Inc., Chicago, IL, USA). Total 20 patients were included in statistical analysis since patients number 18 and 21 were excluded due to unstable weight fluctuations (over two kilos change).

Descriptive analysis was performed on the cohort, recording the number of valid cases for each test, minimum and maximum values measured, mean and standard deviation (SD), shown in Table 2.

Weight and BMI category refer to the initial weight and BMI. Weight change and waist's circumference change refer to the measurements' difference between the baselines and post six treatment sessions. Another parameter indicated is the percentage change, referring to each patient's net measurement divided by the initial factor. For example weight percentage change was calculated as the change between the patient's baseline and post six treatment sessions weight measurement divided by the patient's baseline weight measurement.

A linear correlation tests (R squared) were performed between two parameters: circumference change as dependent variable and weight percentage change, age, initial BMI or high as independent variable.

\section{Results}

All twenty two patients treated for six treatments by the bi-polar RF device showed some degree of total circumferential reduction from $-25.0 \mathrm{~cm}$ up to $-1.5 \mathrm{~cm}$ (Table 2). Since the calculation of total circumferential reduction was inconsistent (in some of the patients not all anatomic parts of body where measured), the umbilicus circumference change was chosen as a constant parameter for statistical analysis and comparison. The umbilicus circumference change ranged between the loss of $5 \mathrm{~cm}$ to no change with a mean value of $-2 \mathrm{~cm}(\mathrm{SD}=$ 1.5; Table 2).

In contrary to the circumference change, the weight change was low (Table 2) with a mean value of -0.0720 Kg and mean percentage of $-0.0016 \%$ ( $S D=0.01499$ ), demonstrating stable weight of the cohort.

No adverse events were recorded during the whole period of trial. The side effects were limited to local bruises, which resolved during the next 4 to 9 days post-treatment. Temporary erythema, which accepted as positive end-point, lasted up to 60 minutes post-treatment.

During the 3-month follow up visit, no rebound of the body condition was detected (data not shown).

A linear correlation tests (R squared) between umbilicus's circumference change and weight change \%, did not reveille strong statistical significant relation, with $\mathrm{R}^{2}$ of 0.07 and sig $=0.260$.

Also, there was no statistical significant relation between the umbilicus's circumference change and the age $\left(\mathrm{R}^{2}=0.09\right.$, sig $\left.=0.299\right)$, height $\left(\mathrm{R}^{2}=0.17\right.$, sig $\left.=0.409\right)$ or initial BMI of the patient $\left(\mathrm{R}^{2}=0.20\right.$, sig $\left.=0.443\right)$.

\begin{tabular}{cccccc} 
Table 2. Descriptive statistics. & & & & \\
\hline Parameter & N & Minimum & Maximum & Mean & Std. Deviation \\
\hline Age & 20 & 24 & 51 & 39.50 & 7.280 \\
Weight (Kg) & 20 & 49.50 & 80.90 & 61.5185 & 9.19776 \\
BMI & 20 & 18.78 & 28.41 & 22.3478 & 2.61494 \\
Weight change (Kg) & 20 & -2.00 & 1.86 & -0.0720 & 0.84627 \\
Weight change \% & 20 & -0.04 & 0.03 & -0.0016 & 0.01499 \\
Total circumference change (cm) & 20 & -25.0 & -1.5 & -10.150 & 5.9096 \\
Umbilicus's circumference change (cm) & 20 & -5.00 & 0.00 & -2.0000 & 1.46898 \\
\hline
\end{tabular}




\section{Discussion and Conclusions}

This is well known that body shape and body image are negatively impacted by unwanted local subcutaneous fat. Therefore body contouring treatments are increasingly popular cosmetic procedures. Thermal stimulus by non-invasive systems can increase physiological breakdown of triglycerides within adipocytes. Besides, recent animal and human studies showed there was macrophage infiltration and delayed adipocytes cellular death or Apoptosis after an adipose tissue was exposed to therapeutic temperature at $43^{\circ} \mathrm{C}$ to $45^{\circ} \mathrm{C}$ for 15 minutes. Consequently, shrinkage of subcutaneous fat layer was resulted [13]-[14].

Most RF-based systems aimed for body shaping and contouring report $2-4 \mathrm{~cm}$ circumferential reductions in abdominal-hip measurements, and usually happy patients [2]-[8] [15]. Therefore it is not surprisingly that in current study the majority of the patients exhibited a substantial circumferential reduction, not at the expense of weight loss.

Two of the patients (patient No. 3 and 13) did not exhibit any circumferential reduction, according to the umbilicus measurements, but did show substantial circumferential reduction of the hip and tights (lower body). Here it is interesting to speculate about concept called redistribution of the fat, due to "fat communication" hormones or peptides. That is why when abdominal subcutaneous fat is reduced or influenced, measurement of other areas like frank, thighs, arms, even neck may also be reduced subsequently. View of the aforesaid, it is quite interesting to investigate this area in more scientific framework.

The lack of statistically significant correlation between umbilicus's circumference change and weight change, portraits the independent relation between the two, leaving the impressive loss of patients waists' circumferential reduction be the solely result of the treatment's course. The thermal stimulus by RF energy to the superficial adipose tissue, enhance the natural lipolysis.

When randomly selected six of the enrolled patients received additional series of treatment sessions (more than six sessions), the circumferential reduction further accelerated (data not shown). These patients exhibited up to 3 times more circumferential reduction reduced, compared to the achieved reduction after the first six treatment sessions (see Example in Figure 1). For example, patient No. 2 reduced only $0.5 \mathrm{~cm}$ according to umbilicus's measurements after the first six treatment sessions and additional $1.5 \mathrm{~cm}$ after additional six sessions (total-2 cm post 12 treatment sessions). This acceleration of clinical outcomes can be contributed to enhanced metabolic rate of the adipose tissue. This type of "buildup" in circumferential reduction can be assumed when the initial blood microcirculation in the treatment area is poor. While increasing the blood flow by the thermal influence of RF energy, every consecutive treatment will lead to greater physiological response, due to increase in skin conductivity and the ability to reach higher end point temperature during the treatment [11].

Three of the patients enrolled in currents study were also treated on additional body areas, including underarms, hips and thighs with notable improvement in circumferential reduction (Table 3). Due to very small sample size of the additional areas, only abdomen area was chosen for statistical analysis. In addition, abdominal measurements were the most consistent, while other areas were highly variable and differentiated in measurement (some measurements were missing or taken in different locations on the body or position) which affected the variability of the patients.

Although no significant correlation was found between the circumferential measurements and initial patients parameters, it could have been interesting to check the correlation between the clinical outcomes and the patient's gender due to physiological/metabolic differences between the genders. In women, lipid changes occur more slowly within the femoral region than within the abdominal region; the adipocytes within the femoral region are

Table 3. Circumferential reduction on additional body areas.
\begin{tabular}{cccc}
\hline Circumferential Reduction $(\mathrm{cm})$ & Patient No. 3 & Patient No. 8 & Patient No. 17 \\
\hline Hip & -5.50 & -3.00 & NA \\
Right thigh & -2.50 & NA & -2.50 \\
Left thigh & -1.50 & NA & -2.50 \\
Right arm & NA & -1.50 & NA \\
Left arm & NA & -1.50 & NA \\
\hline
\end{tabular}

NA: Not Available. 


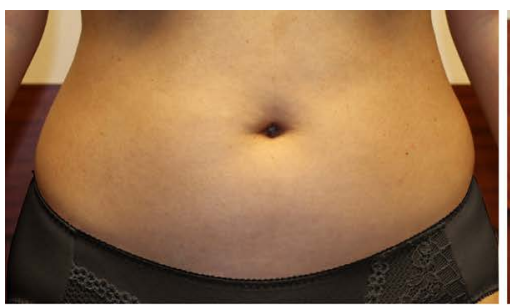

Before

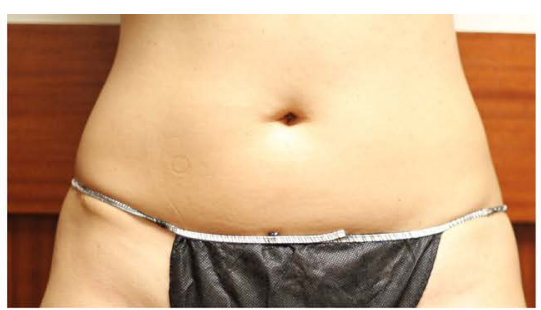

After 6 Treatments

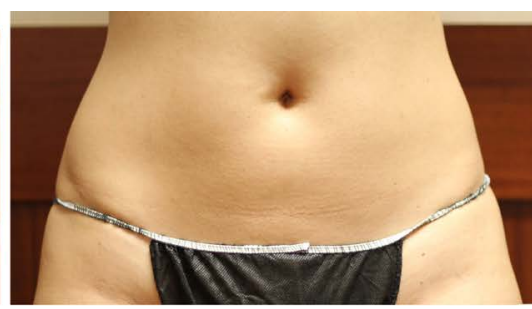

After 12 Treatments

Figure 1. 38-year-old female patient, before, after 6 and 12 abdomen contouring treatments with bi-polar RF device.

larger and are influenced by female sex hormones. They are metabolically more stable and resistant to lipolysis [13]. But, since the male group in this study was relatively low (only 6 males), statistical examination of this theory was impossible.

Safety issues are main concern in performing aesthetic procedures. This non-invasive bi-polar RF-based system delivers therapeutic thermal energy selectively to subcutaneous adipose tissues without causing damages to surrounding epidermis, dermis and muscles.

Since RF energy, in contrary to light energy, produces an electrical current, epidermal melanin reminds unscathed, making the RF-based devices as a safe choice for patients of all skin types.

Facial aging are resulted from combination effects of gravity, reduced skin elasticity, rearrangement of subcutaneous volume, change in supporting bony structure and etc. There may be areas of fat accumulation such as at lower face, submental and submandibular regions, which are particularly common in aging Asians.

Recently, the safety and efficacy of the system was also evaluated on Asian patients with facial aging and local facial fat accumulation (data not shown). A group of 15 participants underwent a treatment course of 4 - 8 sessions and showed notable facial skin tightening, upward repositioning of facial volume and reduction in excessive fat accumulation at lower face and neck regions.

In conclusion, the bi-polar RF system, which is a bi-polar RF system utilizing multiple frequencies of RF together with vacuum associated mechanical pressure, is a safe and effective treatment option of non-invasive body contouring and localized fat reduction among Asian population.

\section{References}

[1] Sadick, N.S. and Mulholland, R.S. (2004) A Prospective Clinical Study to Evaluate the Efficacy and Safety of Cellulite Treatment Using the Combination of Optical and RF Energies for Subcutaneous Tissue Heating. Journal of Cosmetic and Laser Therapy, 6, 187-190. http://dx.doi.org/10.1080/14764170410003039

[2] Alster, T.S. and Tanzi, E.L. (2005) Cellulite Treatment Using a Novel Combination Radiofrequency, Infrared Light, and Mechanical Tissue Manipulation Device. Journal of Cosmetic and Laser Therapy, 7, 81-85. http://dx.doi.org/10.1080/14764170500190242

[3] Zachary, C.B., Mian, A. and England, L.J. (2009) Effects of Monopolar Radiofrequency on the Subcutaneous Fat Layer in an Animal Model [Abstracts]. American Society for Laser Medicine and Surgery, 38, 105.

[4] Anolik, R., Chapas, A.M., Brightman, L.A. and Geronemus, R.G. (2009) Radiofrequency Devices for Body Shaping: A Review and Study of 12 Patients. Seminars in Cutaneous Medicine and Surgery, 28, 236-243.

[5] Goldberg, D.J., Fazeli, A. and Berlin, A.L. (2008) Clinical, Laboratory, and MRI Analysis of Cellulite Treatment with a Unipolar Radiofrequency Device. Dermatologic Surgery, 34, 204-209. http://dx.doi.org/10.1097/00042728-200802000-00009

[6] Emilia del Pino, M., Rosado, R.H., Azuela, A., Graciela Guzmán, M., Argüelles, D., et al. (2006) Effect of Controlled Volumetric Tissue Heating with Radiofrequency on Cellulite and the Subcutaneous Tissue of the Buttocks and Thighs. Journal of Drugs in Dermatology, 5, 714-722.

[7] Kaplan, H. and Gat, A. (2009) Clinical and Histopathological Results Following TriPollar Radiofrequency Skin Treatments. Journal of Cosmetic and Laser Therapy, 11, 78-84. http://dx.doi.org/10.1080/14764170902846227

[8] Teitelbaum, S.A., Burns, J.L., Kubota, J., Matsuda, H., Otto, M.J., et al. (2007) Noninvasive Body Contouring by focused Ultrasound: Safety and Efficacy of the Contour I Device in a Multicenter, Controlled, Clinical Study. Plastic and Reconstructive Surgery, 120, 779-789. http://dx.doi.org/10.1097/01.prs.0000270840.98133.c8

[9] Shek, S.Y., Chan, N.P. and Chan, H.H. (2012) Non-Invasive Cryolipolysis for Body Contouring in Chinese-A First Commercial Experience. Lasers in Surgery and Medicine, 44, 125-130. http://dx.doi.org/10.1002/lsm.21145 
[10] McRae, E. and Boris, J. (2013) Independent Evaluation of Low-Level Laser Therapy at 635 nm for Non-Invasive Body Contouring of the Waist, Hips, and Thighs. Lasers in Surgery and Medicine, 45, 1-7. http://dx.doi.org/10.1002/lsm.22113

[11] Belenky, I., Margulis, A., Elman, M., Bar-Yosef, U. and Paun, S.D. (2012) Exploring Channeling Optimized Radiofrequency Energy: A Review of Radiofrequency History and Applications in Esthetic Fields. Advances in Therapy, 29, 249-266. http://dx.doi.org/10.1007/s12325-012-0004-1

[12] Chan, H.H.L. (2005) Effective and Safe Use of Lasers, Light Sources, and Radiofrequency Devices in the Clinical Management of Asian Patients with Selected Dermatoses. Lasers in Surgery and Medicine, 37, 179-185. http://dx.doi.org/10.1002/lsm.20244

[13] Franco, W., Kothare, A., Ronan, S.J., Grekin, R.C. and McCalmont, T.H. (2010) Hyperthermic Injury to Adipocyte cells by Selective Heating of Subcutaneous Fat with A Novel Radiofrequency Device: Feasibility Studies. Lasers in Surgery and Medicine, 42, 361-370. http://dx.doi.org/10.1002/lsm.20925

[14] Weiss, R., Weiss, M., Beasley, K., Vrba, J. and Bernardy, J. (2013) Operator Independent Focused High Frequency ISM Band for Fat Reduction: Porcine model. Lasers in Surgery and Medicine, 45, 235-239. http://dx.doi.org/10.1002/lsm.22134

[15] Brightman, L., Weiss, E., Chapas, A.M., Karen, J., Hale, E., et al. (2009) Improvement in Arm and Post-Partum Abdominal and Flank Subcutaneous Fat Deposits and Skin Laxity Using a Bipolar Radiofrequency, Infrared, Vacuum and Mechanical Massage Device. Lasers in Surgery and Medicine, 41, 791-798. http://dx.doi.org/10.1002/lsm.20872 